\title{
Digital phenotyping of complex psychological responses to the COVID-19 pandemic
}

\author{
Thomas D. Hull \\ Talkspace \\ Jacob Levine \\ Talkspace \\ Niels Bantilan \\ Talkspace \\ Angel N. Desai \\ University of California, Davis \\ Maimuna S. Majumder \\ Boston Children's Hospital, Harvard Medical School
}




\begin{abstract}
Background: The novel coronavirus disease 2019 (COVID-19) has negatively impacted mortality, economic conditions, and mental health. A large scale study on psychological reactions to the pandemic to inform ongoing population-level symptom tracking and response to treatment is currently lacking.
\end{abstract}

Methods: Average intake scores for standard depression and anxiety symptom scales were tracked from January 1, 2017 to June 9, 2020 for patients seeking treatment from a digital mental health service to gauge the relationship between COVID-19 and self-reported symptoms. We applied natural language processing (NLP) to unstructured therapy transcript data from patients seeking treatment during the height of the pandemic in the United States between March 1, 2020 and June 9, 2020 to identify words associated with COVID-19 mentions. This analysis was used to identify symptoms that were present beyond those assessed by standard depression and anxiety measures.

Results: Depression and anxiety symptoms reported by 169,889 patients between January 1, 2017 and June 9, 2020 were identified. There was no detectable change in intake depression symptom scores. Intake anxiety symptom scores increased 1.42 scale points [95\% CI: 1.18, 1.65] between March 15, 2020 and April 1, 2020, when scores peaked. In the transcript data of these 169,889 patients, plus an expanded sample of 49,267 patients without symptom reports, term frequency-inverse document frequency (tf-idf) identified 2,377 positively correlated and 661 
negatively correlated terms that were significantly $(F D R<.01)$ associated with mentions of the virus. These terms were classifiable into 24 symptoms beyond those included in the diagnostic criteria for anxiety or depression.

Conclusions: The COVID-19 pandemic may have increased intake anxiety symptoms for individuals seeking digital mental health treatment. NLP analyses suggest that standard symptom scales for depression and anxiety alone are inadequate to fully assess and track psychological reactions to the pandemic. Symptoms of grief, trauma, obsession-compulsion, agoraphobia, hypochondriasis, panic, and nonsuicidal self-injury should be monitored as part of a new COVID-19 Syndrome category.

Keywords: digital phenotyping, COVID-19, psychological sequalae, telehealth, digital mental health, natural language processing, machine learning 


\section{Introduction}

Since late 2019, the ongoing novel coronavirus disease 2019 (COVID-19) pandemic has proved to be extremely disruptive with high morbidity and mortality, as well as economic and mental health consequences. This poses a challenge for mental health services given how little is known about the virus and the psychological impact this crisis is having on medical professionals, essential workers, individuals facing unemployment, and others observing physical distancing recommendations. Early reports suggest that mental health professionals have faced significant challenges due to lack of information and established guidelines for assessment and treatment ${ }^{1}$. Psychological sequelae to major events like COVID-19 vary greatly ${ }^{2-4}$, making it difficult to assess the full range of symptoms potentially related to the current crisis and to track the course of these reactions over time. The use of comprehensive symptom checklists and exhaustive clinical interviews poses a significant burden to clinicians and respondents alike that could be avoided if the most common symptoms were known ahead of time.

One major obstacle to identifying the most relevant symptoms for screening is the amount of time it takes to amass clinical observations of a suitably large patient population in order to determine the full scope of patient reactions. It is difficult to differentiate peripheral symptoms from those that are more central and pathogenic ${ }^{5}$ ) and to differentiate symptoms that would ordinarily be reported by a diverse treatment-seeking population from those more closely associated with COVID-19-related concerns. This type of data is currently lacking for the COVID-19 crisis. Data generated by large telemental health services that deliver care remotely 
in the United States and globally may be helpful in describing the full complexity of patient clinical presentations. The richest form of these data is the textual exchanges made between patients and therapists as part of intake and treatment procedures. These data are relatively unstructured in comparison to standardized symptom measures but offer the advantage of capturing the patient's experience more comprehensively, allowing for separation of symptoms reported in conjunction with mentions of COVID-19 from symptoms reported by individuals seeking care for other reasons. A broad set of methods referred to as natural language processing (NLP) have been designed for working with unstructured textual data. They range from simple methods that search for specific words in a block of text to more complex neural network models that aim to extract the meaning of certain statements from within the larger context of a text corpus.

The aim of this study is to identify major themes and symptom clusters in the messages sent by patients to therapists while seeking treatment for pandemicrelated distress on a popular telemental health platform, Talkspace. First, we separate symptoms associated with the pandemic from symptoms otherwise experienced by individuals seeking treatment by investigating relationships between words associated with COVID-19 mentions. This is impossible to achieve when relying on structured symptom measures alone that do not specify whether symptoms relate to the pandemic or to some other cause. We describe a multistep process that employs brief symptom measures to determine the relationship between the pandemic and common self-reported anxiety and depression symptoms, identifies patients who expressed COVID-19-related concerns, and 
isolates words that are highly correlated with mentions of the virus. These associated words are categorized using a digital phenotyping process that counts the prevalence of clinical and nonclinical themes to ultimately identify symptoms from diagnostic categories that are not reflected in structured measures of common anxiety and depression symptoms. We demonstrate that this method has strong face validity and clinical interpretability and could potentially inform the choice of structured measures for tracking responses to the pandemic over time. To this end, we propose COVID-19 Syndrome as a clinical category derived through machine identification of symptoms.

\section{Methods}

Setting

Talkspace is a telemental health platform that enables licensed psychotherapists to deliver care through asynchronous two-way messaging including text messages, audio messages, video messages, and by scheduled live video sessions to patients within their regions of licensure. It has demonstrated acceptability and feasibility for increasing access to care ${ }^{6}$ and is in use by over two thousand therapists who serve fifteen to twenty patients each on average at any given time throughout the United States and globally. The platform enables seamless integration of symptom and outcome measures, as well as crisis and referral services for those in need of higher levels of care.

\section{Participants}


To evaluate changes in self-reported symptoms, the seven-item Generalized Anxiety Disorder questionnaire ${ }^{7}$ and the nine-item Patient Health Questionnaire ${ }^{8}$ were administered to all patients initiating treatment for depression and/or anxiety between January 1, 2017 and June 9, 2020 allowing for the comparison of historical trends in self-reported symptoms against those that are contemporaneous to the ongoing COVID-19 pandemic.

To evaluate symptoms not included in standard measures of depression and anxiety, regular expressions (i.e., regex) related to the pandemic were generated, including "corona," "virus," "covid," and "pandemic." All expressions were verified to have near 0\% incidence prior to February 2020 (see Table 1). Using computerized keyword matching, all patient messages included in treatment transcripts that were generated between March 1, 2020 and June 9, 2020 were analyzed to identify COVID-19-related terms. We define a "transcript" as the set of all messages exchanged between a patient and a provider, such that each patient has exactly one transcript.

[Table 1 about here]

Statistical Analysis

Average summary scores for both anxiety and depression scales were constructed, aggregated, and anchored by the patient's day of admission to assess change over time. Analyses were conducted with the Python programming language, using the following packages for statistical analysis: pandas for data 
analysis $^{9,10}$, matplotlib $^{11}$, and seaborn ${ }^{12}$ for visualization, and scipy ${ }^{13}$, and pandera ${ }^{14}$ for data validation and hypothesis testing.

In order to isolate the words and phrases (i.e., n-grams) most likely to appear with COVID-19-related mentions, each day of text in this period was represented using natural language processing (NLP) as a vector of word counts and transformed by term frequency-inverse document frequency (tf-idf), which in this setting served to emphasize words that changed in frequency over time. The Pearson correlation coefficient was computed between each word's tf-idf trajectory and the proportion of messages containing COVID-19-related mentions during the same period. Only terms that were below a threshold of 0.01 for the false discovery rate (FDR) were selected. Analyses were conducted with the Python programming language using spacy ${ }^{15}$ and textacy ${ }^{16}$ for NLP analyses, and scipy ${ }^{13}$ and statsmodels ${ }^{17}$ for statistical analyses.

Since the resulting word lists have no identifiable structure on their own, these words were assigned to empirically-derived, human-validated topics using Empath $^{18}$ - a software program that assigns words to topical categories based on similarities in word use (i.e., word embeddings) that are then validated by human curators. The percentage of words in each topical category assigned by Empath were counted.

Empath categories are a non-arbitrary method for evaluating major topics associated with mentions of COVID-19 - including positive and negative emotion states - but do not categorize words in relation to diagnostic criteria. To determine the relationship between words associated with mentions of COVID-19 and 
diagnostic categories, these words were converted into a dictionary for search against the publicly available International Classification of Diseases, Tenth Revision (ICD-10) symptom descriptions for the classification of mental and behavioral disorders. Words and phrases that did not match existing diagnostic criteria were inspected for clinical relevance and reported as additional criteria.

All data were analyzed using machine analytics and were de-identified prior to analysis. Patients using the service provide consent to the use of their data in an aggregated and de-identified format for research purposes. Procedures for the collection of symptom questionnaires were approved by the Teachers College, Columbia University IRB (15-426).

\section{Results}

Participants

We identified symptom data for 169,889 patients between January 1, 2017 and June 9, 2020. The largest age group was 26-35 years (55\% of the sample). Women accounted for $73.2 \%(124,358)$ of the included participants. A total of $60.3 \%$ of participants $(102,443)$ identified as European American. There were at minimum 2,211 patients from every state (with the largest numbers being from California, 24,634 patients (14.5\% of the total), and New York, 20,387 patients $(12 \%))$. Nearly forty-five percent $(44.6 \%, 75,770)$ of participants reported being in therapy for the first time (see Table 2).

[Table 2 here] 
Outcomes

Intake averages for the GAD-7 showed a 1.42 point increase $(95 \%$ CI $[1.18$, 1.65]) in the average intake severity of anxiety symptoms for the 10,645 patients presenting for depression and/or anxiety treatment between March 15, 2020 and April 1, 2020, when scores were at their highest peak. There is an ongoing 0.33 point increase $(95 \%$ CI $[.11, .54])$ in GAD-7 scores as of June 9, 2020 (see Figure 1, Figure 2). No significant change was observed in PHQ-9 scores for intake depression severity.

[Figure 1 about here]

A total of 219,156 transcripts were identified and included the 169,889 patients for whom outcome data was available. The remaining 49,267 patients chose not to complete an intake assessment or their response was incomplete. All transcripts were analyzed and 18.5\% (40,448 transcripts) were found to contain coronavirus mentions. Of the 500,000 words and phrases examined from the transcripts with COVID-19 mentions, we identified 2,377 positively correlated and 661 negatively correlated terms that were significantly associated with mentions of the virus. The words that were most highly correlated with COVID-19-related mentions demonstrate a set of complex reactions that include the Empath categories of confusion and negative emotion (30\% of assigned words); health and medical emergencies (21\% of assigned words); work, business, and economic concerns (18\% of assigned words ); technology and internet (12\% of assigned 
words); cleaning and hygiene (8\% of assigned words); government and leadership (7\% of assigned words); and traveling and shopping (4\% of assigned words). Empath categories negatively correlated with COVID-19 mentions were party and celebration (30\% of assigned words); positive emotion and love $(27 \%$ of assigned words); friends and children ( $27 \%$ of assigned words); and optimism ( $16 \%$ of assigned words). While words associated with parties, celebrations, love, friends and children were far less likely to co-occur with COVID-19 mentions, the wordstem "lone," (which would include "alone," "lonely," and "loneliness," etc.) was not significantly related to pandemic concerns.

Words and phrases for these Empath categories exhibited similarities to existing ICD-10 diagnostic classifications including acute stress reaction (ICD-10 F43.0), which would meet criteria for a trauma reaction (ICD-10 F43.1) if persistent over time; paranoia (ICD-10 F22); grief (ICD-10 Z63.4), though symptom persistence over 6 months is needed to meet full criteria); insomnia (ICD-10 G47.00); panic (ICD-10 F41.0); agoraphobia (ICD-10 F40.00); non-suicidal self injury (ICD-10 Z91.5); obsession-compulsion (ICD-10 F42.9); and hypochondriasis (ICD-10 F45.21) symptoms. Additional clinically relevant content included confusion about one's state and difficulty controlling anger at others and institutions (see Table 3).

[Table 3 here]

\section{Discussion}

In this study, we investigated the relationship between the COVID-19 pandemic and intake anxiety and depression symptoms for treatment-seeking 
patients on a digital mental health platform. A significant and noticeable increase was identified for anxiety symptoms, but not for depression symptoms. We also applied machine learning methods to a large body of treatment transcripts via NLP to identify additional symptoms associated with mentions of COVID-19, which would have been missed by symptom measures assessing anxiety or depression alone. These additional symptoms include those associated with other diagnostic categories such as acute stress, PTSD, grief, OCD, insomnia, hypochondriasis, nonsuicidal self-injury, and paranoia. A more complex symptom profile is in some ways anticipated by dimensional approaches to psychopathologic nosology ${ }^{19,20}$ that focus more on symptoms and functioning than on diagnostic categories. Our work suggests that tracking the lasting psychological impact of COVID-19 will require measures that include a variety of symptoms from several disorders. Survey-based studies to date have accounted for a mix of depression, anxiety, insomnia, and stress-related conditions ${ }^{21-23}$, but have not reported data on the other syndromes we have identified here. Constructing an appropriate measure - whether by combining self-reporting with clinician or other ratings, utilizing advantages of ecological momentary assessment, or other strategies for mitigating recall bias - is beyond the scope of this study, but we highlight this as an important next step in translating our findings to practice.

An assessment composed of symptom questions informed by the analyses above could ultimately identify subpopulations of patients with different symptom profiles to assist in individualizing treatment and tracking of heterogeneous responses to clinical intervention. For example, social isolation and loneliness are 
distinct risk pathways for suicide and benefit from being assessed as distinct behaviors to inform treatment planning ${ }^{24}$. This is also the case for individuals with comorbid psychiatric disorders. Comorbidity is common, yet without pandemicspecific longitudinal assessment, true comorbidity could be conflated with changing symptom constellations for the same underlying pathology, as exemplified in the dynamic interplay of bipolar disorder and anxiety symptoms ${ }^{25}$. Selecting symptom questions through the rapid digital phenotyping methods implemented here can help reduce the burden on respondents while enabling a broader dimensional approach to monitoring psychopathology that focuses on symptoms rather than diagnostic categories ${ }^{19}$. Indeed, a focus at the National Institute of Mental Health has been on approaching disorders in a dimensional way that does not rely on disorder categories, but instead draws on "big data" - or large clinical datasets - to understand underlying mechanisms of health and illness ${ }^{26,27}$. The data reported here are an important first step in the effort to understand symptom clusters associated with the pandemic to eventually guide the discovery of pathogenic mechanisms and inform personalized interventions that maximize treatment benefit ${ }^{28-30}$.

To encapsulate the range of clinical observations derived from these analyses, we propose COVID-19 Syndrome as a clinical category to help distinguish pandemic-related clinical presentations from those related to other existing and familiar clinical categories. COVID-19 Syndrome is composed of criteria from other categories, as well as criteria from these analyses. What sets it apart from other clinical categories is the likelihood with which disparate symptoms occur together 
and the difficulty in determining whether responses are a transient reaction to the pandemic or are in need of clinical care.

The lack of "lone" terms may correspond to studies reporting that loneliness is unlikely to be a major factor in the psychological distress of the pandemic ${ }^{23}$, or it could suggest that people are in fact isolated from others, given the far fewer mentions of social topics for those with COVID-19-related complaints in these data, but are not yet thinking of it as "loneliness." The $18.5 \%$ of patients who mentioned the pandemic constitute a substantial increase in disease burden over and above the pre-study patient population already presenting for treatment and is matched by the increase in new cases reported for this platform. An important feature of this study is in distinguishing and quantifying those patients seeking care for COVID-19related concerns versus those who would have sought care without the stressors of a pandemic. While anxiety symptoms began returning to historical levels between May 5, 2020 and May 30, 2020, early June 2020 exhibited a reversal of this trend. It is thus advisable to place continued emphasis on those who experience psychological symptoms and require treatment as a result of the pandemic.

Though this study offers a novel method and dataset for rapidly phenotyping COVID-19-related symptoms, it is not without limitations. First, these results may not generalize beyond the population of individuals who seek treatment through digital platforms. Second, analyses relied on the longitudinal data of a convenience sample that self-reported their symptoms, without any manipulation of referral source or random assignment. Of particular note is the large number of women represented in the sample, though this is consistent with data for the use of 
telemedicine in routine care ${ }^{31}$. Despite these limitations, our results demonstrate the utility of large, unstructured data for rapid digital phenotyping to identify psychological symptoms that are associated with patient reports of COVID-19related concerns but are missed by standard depression and anxiety screeners.

Acknowledgments: Hull, Levine, and Bantilan are employees of Talkspace. Desai and Majumder declare no conflicts. 


\section{References}

1. Xiang $Y T$, Jin $Y$, Cheung $T$. Joint international collaboration to combat mental health challenges during the coronavirus disease 2019 pandemic. JAMA psychiatry. $2020 \mathrm{Apr}$ 10.

2. Meredith LS, Eisenman DP, Tanielian T, Taylor SL, Basurto-Davila R, Zazzali J, Diamond D, Cienfuegos B, Shields S. Prioritizing "psychological" consequences for disaster preparedness and response: a framework for addressing the emotional, behavioral, and cognitive effects of patient surge in large-scale disasters. Disaster medicine and public health preparedness. 2011 Mar;5(1):73-80.

3. Shultz, J.M., Baingana, F, Neria, Y. The 2014 Ebola outbreak and mental health: current status and recommended response. JAMA. 2015;313(6):567- 568.

doi:10.1001/jama.2014.17934

4. Shanafelt T, Ripp J, Trockel M. Understanding and Addressing Sources of Anxiety Among Health Care Professionals During the COVID-19 Pandemic. JAMA. 2020 Apr 7.

5. Holmes EA, O'Connor RC, Perry VH, Tracey I, Wessely S, Arseneault L, Ballard C, Christensen H, Silver RC, Everall I, Ford T. Multidisciplinary research priorities for the COVID-19 pandemic: a call for action for mental health science. The Lancet Psychiatry. 2020 Apr 15.

6. Hull TD, Malgaroli M, Connolly PS, Feuerstein S, Simon NM. Two-way messaging therapy for depression and anxiety: longitudinal response trajectories. BMC Psychiatry. 2020 Dec;20(1):1-2.

7. Spitzer, RL, Kroenke, K, Williams, JBW, \& Lowe, B. (2001). Validity of a brief depression severity measure. Journal of General Internal Medicine, 16, 606-613.

8. Spitzer RL, Kroenke K, Williams JBW, Lowe B. (2006). A brief measure for assessing generalized anxiety disorder. Arch Intern Med, 166:1092-1097.

9. McKinney W. Data structures for statistical computing in python. In Proceedings of the 9th Python in Science Conference 2010 Jun 28 (Vol. 445, pp. 51-56).

10. Reback J, McKinney, W, Brockmendel, J, Bossche, JV, Augspurger, T, Cloud, P, ... Mehyar, M. (2020, March 18). pandas-dev/pandas: Pandas 1.0.3 (Version v1.0.3). Zenodo. http://doi.org/10.5281/zenodo.3715232

11. Hunter JD. Matplotlib: A 2D graphics environment. Computing in science \& engineering. 2007 May;9(3):90-5.

12. Waskom, M, Botvinnik, O, Ostblom, J, Gelbart, M, Lukauskas, $S$, Hobson, $P, \ldots$ Brian. (2020, April 26). mwaskom/seaborn: v0.10.1 (April 2020) (Version v0.10.1). Zenodo. http://doi.org/10.5281/zenodo.3767070

13. Virtanen P, Gommers R, Oliphant TE, Haberland M, Reddy T, Cournapeau D, Burovski E, Peterson P, Weckesser W, Bright J, van der Walt SJ. SciPy 1.0: fundamental algorithms for scientific computing in Python. Nature methods. 2020 Mar;17(3):261-72. 
14. Bantilan, N, Markey, N, Albertazzi, R, chr1st1ank, Kim, S, Lawrence, Z. (2020, June 5). pandera-dev/pandera: bugfix: conda build failure, use version.py file (Version 0.4.2). Zenodo. http://doi.org/10.5281/zenodo.3878775

15. Honnibal, M, Montani, I. (2017). spaCy: Natural language understanding with Bloom embeddings, convolutional neural networks and incremental parsing. http://spacy.io

16. DeWilde, B. (2020). Textacy: NLP, before and after spaCy. http://chartbeatlabs.github.io/textacy

17. Seabold S, Perktold J. Statsmodels: Econometric and statistical modeling with python. In Proceedings of the 9th Python in Science Conference 2010 Jun 28 (Vol. 57, p. 61).

18. Fast E, Chen B, Bernstein MS. Empath: Understanding topic signals in large-scale text. In Proceedings of the $2016 \mathrm{CHI}$ Conference on Human Factors in Computing Systems 2016 May 7 (pp. 4647-4657).

19. Caspi A, Houts RM, Ambler A, Danese A, Elliott ML, Hariri A, Harrington H, Hogan S, Poulton R, Ramrakha S, Rasmussen LJ. Longitudinal Assessment of Mental Health Disorders and Comorbidities Across 4 Decades Among Participants in the Dunedin Birth Cohort Study. JAMA Network Open. 2020 Apr 1;3(4):e203221-.

20. Cuthbert BN. The RDoC framework: facilitating transition from ICD/DSM to dimensional approaches that integrate neuroscience and psychopathology. World Psychiatry. 2014 Feb;13(1):28-35.

21. The Academy of Medical Sciences. 2020. http://www.acmedsci.ac.uk/COVIDmentalhealthsurveys (accessed May 19, 2020).

22. Liu S, Yang L, Zhang C, Xiang YT, Liu Z, Hu S, Zhang B. Online mental health services in China during the COVID-19 outbreak. The Lancet Psychiatry. 2020 Apr 1;7(4):e17-8.

23. McGinty EE, Presskreischer R, Han H, Barry CL. Psychological Distress and Loneliness Reported by US Adults in 2018 and April 2020. JAMA. 2020 Jun 3.

24. O'Connor RC, Kirtley OJ. The integrated motivational-volitional model of suicidal behaviour. Philos Trans R Soc Lond B Biol Sci 2018; 373: 20170268.

25. Pavlova B, Perlis RH, Alda M, Uher R (2015): Lifetime prevalence of anxiety disorders in people with bipolar disorder: A systematic review and meta-analysis. Lancet Psychiatry 2:710-717.

26. Insel TR, Cuthbert BN (2015): Brain disorders? Precisely. Science. 348: 499-500.

27. Frank M, Barch DM, Kurth-Nelson Z, O'Doherty JP, Denève S, Durstewitz D, Krystal JH, Anticevic A, Murray J, Glahn D, Driesen N. Computational psychiatry: New perspectives on mental illness. MIT Press; 2016 Dec 12.

28. Carl E, Witcraft SM, Kauffman BY, Gillespie EM, Becker ES, Cuijpers P, Van Ameringen $\mathrm{M}$, Smits JA, Powers MB. Psychological and pharmacological treatments for generalized anxiety disorder (GAD): a meta-analysis of randomized controlled trials. Cognitive Behaviour Therapy. 2020 Jan 2;49(1):1-21.

29. Hawton K, Witt KG, Salisbury TL, Arensman E, Gunnell D, Hazell P, Townsend E, van Heeringen K. Psychosocial interventions following self-harm in adults: a systematic review and meta-analysis. The Lancet Psychiatry. 2016 Aug 1;3(8):740-50. 
30. Cuijpers P, Cristea IA, Karyotaki E, Reijnders M, Hollon SD. Component studies of psychological treatments of adult depression: a systematic review and meta-analysis. Psychother Res 2019;29: 15-29.

31. Titov, N., Dear, B. F., Staples, L. G., Bennett-Levy, J., Klein, B., Rapee, R. M., . . Nielssen, O. B. The first 30 months of the MindSpot Clinic: Evaluation of a national emental health service against project objectives. Australian and New Zealand Journal of Psychiatry. 2017;51(12): 1227-1239. doi:10.1177/0004867416671598 
Table 1.

Percentage (\%) of Messages Containing Seed Word Regular Expressions Before and After COVID-19.

\begin{tabular}{|r|r|r|r|r|}
\hline Month & corona & virus & covid & pandemic \\
\hline Jan 2020 & $0.0182 \%$ & $0.0683 \%$ & $0.0 \%$ & $0.0017 \%$ \\
\hline Feb 2020 & $0.0785 \%$ & $0.1604 \%$ & $0.0045 \%$ & $0.0074 \%$ \\
\hline Mar 2020 & $1.1113 \%$ & $2.2173 \%$ & $0.9072 \%$ & $0.6105 \%$ \\
\hline Apr 2020 & $0.4743 \%$ & $1.0913 \%$ & $1.2988 \%$ & $1.0372 \%$ \\
\hline May 2020 & $0.2848 \%$ & $0.5751 \%$ & $1.1225 \%$ & $0.9988 \%$ \\
\hline Jun 2020 & $0.2688 \%$ & $0.4727 \%$ & $1.1481 \%$ & $0.9735 \%$ \\
\hline
\end{tabular}

Table 2.

Demographic Characteristics for Full Sample $(\mathrm{N}=169,889)$.

\begin{tabular}{|c|c|c|}
\hline \multicolumn{1}{|c|}{ Variable } & \# Missing & Percentage (N) \\
\hline Age: & 9,082 & $24 \%(38,594)$ \\
\hline $18-25$ & & $55 \%(88,444)$ \\
\hline $26-35$ & & $14.9 \%(23,960)$ \\
\hline $36-49$ & & $6.1 \%(9,809)$ \\
\hline $50+$ & & \\
\hline Education: & & \\
\hline Bachelor Degree or Higher & & $74.9 \%(108,857)$ \\
\hline & & $25.1 \%(36,480)$ \\
\hline High School Diploma & & \\
\hline & & \\
\hline
\end{tabular}




\begin{tabular}{|c|c|c|}
\hline European American & & $60.3 \%(51,222)$ \\
\hline African American & & $16.8 \%(14,271)$ \\
\hline Southeast/Asian American & & $10.0 \%(8,495)$ \\
\hline Native American & & $0.5 \%(425)$ \\
\hline Other & & $12.4 \%(10,533)$ \\
\hline Hispanic/Latinx & & $15.3 \%(12,997)$ \\
\hline Gender: & 2,330 & \\
\hline Female & & $73.2 \%(122,653)$ \\
\hline Male & & $24.1 \%(40,382)$ \\
\hline [other identity] & & $2.7 \%(4,524)$ \\
\hline Patient's State: & 0 & \\
\hline California & & $14.5 \%(24,634)$ \\
\hline New York & & $12.0 \%(20,387)$ \\
\hline Texas & & $7.7 \%(13,081)$ \\
\hline Florida & & $5.4 \%(9,174)$ \\
\hline [other U.S. State] & & $60.4 \%(102,613)$ \\
\hline
\end{tabular}

Figure 1.

Two Week Rolling Averages Comparing February 1, 2020 to June 9, 2020 with the Same Span for Years 2017, 2018, and 2019. 
2020 GAD-7 levels compared to baseline year (two-week rolling average)
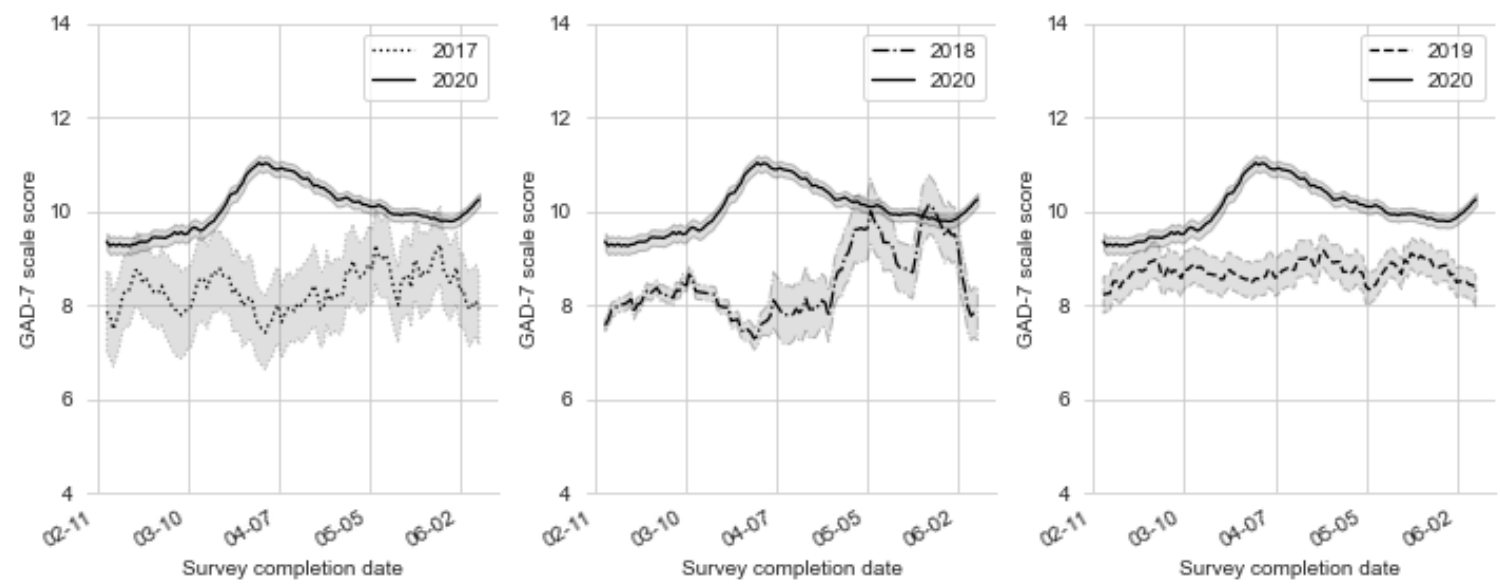

Table 3.

ICD-10 Diagnostic Categories and Symptoms Identified by Digital Phenotyping.

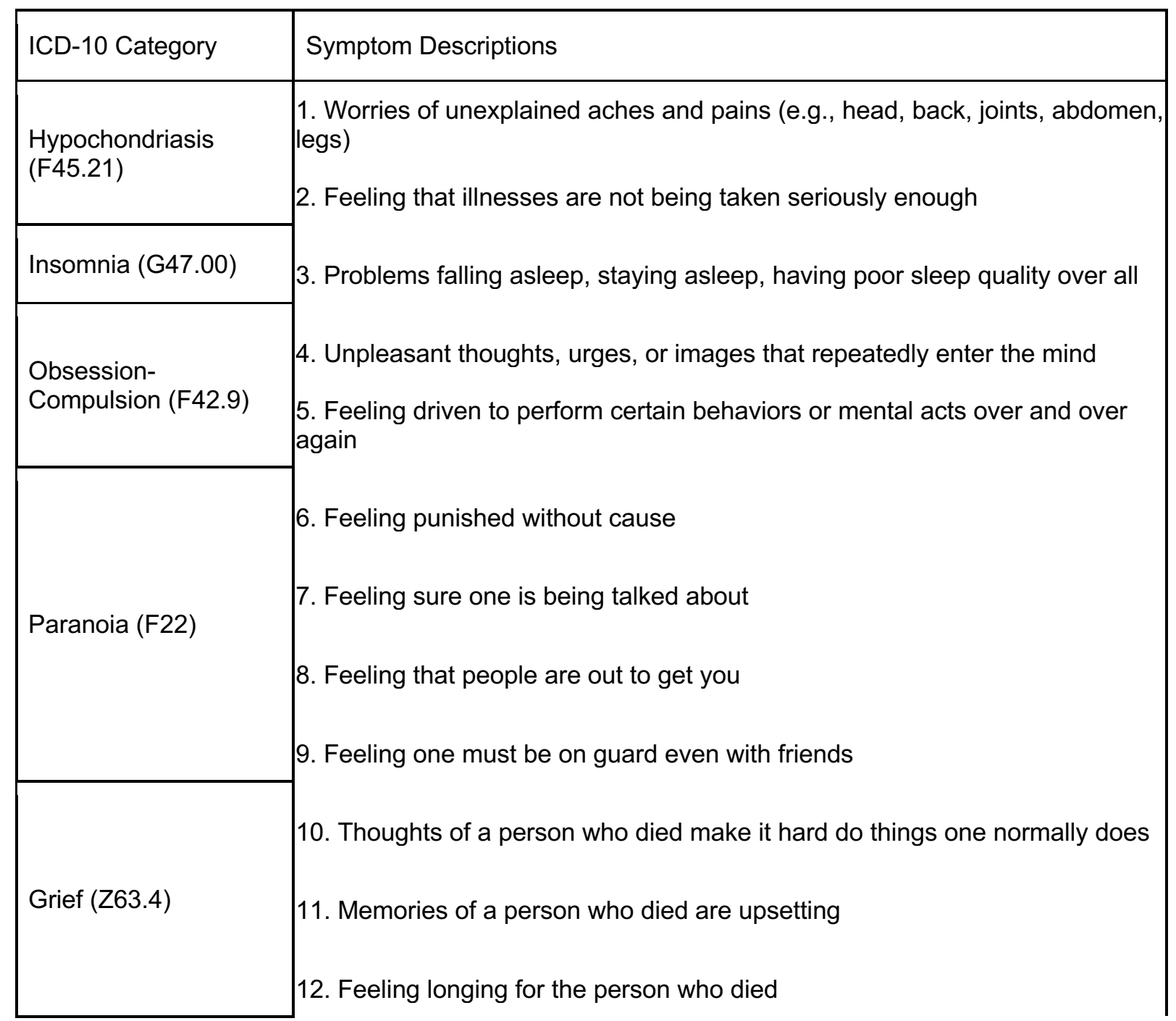




\begin{tabular}{|c|c|}
\hline & 13. Feeling angry about the death \\
\hline $\begin{array}{l}\text { Acute Stress (F43.0); } \\
\text { PTSD (F43.1) }\end{array}$ & $\begin{array}{l}\text { 14. Experiencing an especially frightening, horrible, or traumatic event } \\
\text { 15. Having nightmares about the event(s) or thoughts about the event(s) } \\
\text { when one did not want to } \\
\text { 16. Trying hard not to think about the event(s) or going out of the way to avoid } \\
\text { situations that are reminders of the event(s) } \\
\text { 17. Feeling constantly on guard, watchful, or easily startled } \\
\text { 18. Feeling numb or detached from people, activities, or surroundings } \\
\text { 19. Feeling guilty or unable to stop blaming oneself or others for the event(s) } \\
\text { or any problems the events may have caused }\end{array}$ \\
\hline $\begin{array}{l}\text { Nonsuicidal Self Injury } \\
\text { (Z91.5) }\end{array}$ & $\begin{array}{l}\text { 20. Deliberately hurting oneself physically without intending to kill oneself or } \\
\text { as a strategy for relief }\end{array}$ \\
\hline Panic (F41.0) & $\begin{array}{l}\text { 21. Experiencing panic episodes } \\
\text { 22. Worrying about having another episode }\end{array}$ \\
\hline Agoraphobia (F40.00) & $\begin{array}{l}\text { 23. Worrying about being in a public space in which escape might not be } \\
\text { available should excessive anxiety or panic symptoms develop } \\
\text { 24. Obsessive, persistent, intense fear of open places }\end{array}$ \\
\hline \multicolumn{2}{|c|}{ Anxiety-related symptoms } \\
\hline Anxiety (F41.9) & $\begin{array}{l}\text { 1. Feeling afraid as if something awful might happen } \\
\text { 2. Not being able to stop or control worry } \\
\text { 3. Worrying too much about different things } \\
\text { 4. Becoming easily annoyed or irritable }\end{array}$ \\
\hline \multicolumn{2}{|c|}{ COVID-19 Syndrome specific criteria } \\
\hline $\begin{array}{l}\text { 1. Feeling unsure } \\
\text { 2. Difficulty contro }\end{array}$ & $\begin{array}{l}\text { whether psychological reactions are normative vs. problematic } \\
\text { Illing anger at others' actions (or lack of action) }\end{array}$ \\
\hline
\end{tabular}

